\title{
PRODUCTS OF FULLY COMPLETE SPACES
}

BY W. H. SUMMERS

Communicated by P. Emery Thomas, February 14, 1969

The permanence properties of fully complete spaces were considered first by Collins, and in his paper [1] which appeared in 1955 it is shown that an arbitrary product of fully complete spaces need not be fully complete. In 1958, Kelley [5] introduced the notion of hypercomplete spaces (a priori, fully complete), and conjectured that countable products of hypercomplete spaces would be hyperromplete. The purpose of this paper is to resolve this conjecture.

Lemma 1. If $X$ is a Fréchet space and if $X_{\tau}^{*}$ denotes the topological dual of $X$ endowed with the Mackey topology $\tau\left(X^{*}, X\right)$, then the direct sum $E$ of denumerably many copies of $X_{\tau}^{*}$ has the. Krein-Smulian property (for a definition, see [2, p. 364]), and hence is both hypercomplete and fully complete.

Since the strict topology $\beta$ on $l^{\infty}$ is $\tau\left(l^{\infty}, l^{1}\right)$ from [3], we have the following result.

Lemma 2. The direct sum of denumerably many copies of $l_{\beta}^{\infty}$ has the Krein-Smulian property.

THEOREM 1. If $E$ is the direct sum of denumerably many copies of $l_{\beta}^{\infty}$ and if $F$ is the product of denumerably many copies of $c_{0}\left(c_{0}\right.$ is, of course, endowed with the uniform topology), then both $E$ and $F$ have the Krein-Smulian property, but $E \times F$ is not fully complete.

That $E \times F$ is not fully complete was suggested by an example due to Grothendieck [4, p. 92], and it should be noted that, since a Fréchet space has the Krein-Smulian property, $E \times F$ can be considered as a product of spaces with the Krein-Smulian property of any finite or denumerable order.

We conclude by noting that the question of whether or not $B_{r-}$ completeness is preserved by products is still open.

\section{REFERENCES}

1. H. S. Collins, Completeness and compactness in linear topological spaces, Trans. Amer. Math. Soc. 79 (1955), 256-280.

2. - On the space $l^{\infty}(S)$ with the strict topology, Math. Z. 106 (1968), 361-373.

3. J. B. Conway, The strict topology and compactness in the space of measures, Bull. Amer. Math. Soc. 72 (1966), 75-78.

4. A. Grothendieck, Sur les espaces $(F)$ et $(D F)$, Summa Brasil. Math. 3 (1954), 57-122.

5. J. L. Kelley, Hypercomplete linear topological spaces, Michigan Math. J. 5 (1958), 235-246.

University of Arkansas, Fayetreville, Arkansas 72701 